\title{
A NOTE ON GRADED ALGEBRAS
}

\author{
EDMUND R. PUCZYLOWSKI
}

(Communicated by Donald S. Passman)

\begin{abstract}
It is proved that if an algebra over a field of characteristic $p>0$ is graded by a finite $p$-group, then its ideals having nilpotent intersection with the identity component are nilpotent themselves.
\end{abstract}

In [2] Krempa proved that if $A$ is a prime algebra over a field $F$ of characteristic $p>0$ and $F \subseteq K=F(a)$ is a field extension with $a^{p^{n}} \in F$ for some $n$, then every ideal $I$ of $A \otimes_{F} K$ satisfying $A \cap I=0$ is nilpotent. The proof was based on a use of Martindale's rings of quotients. In this note we give an elementary proof of the following more general result.

Theorem. Let $R$ be a G-graded $F$-algebra, where $G$ is a finite p-group and $F$ is a field of characteristic $p>0$. If $I$ is an ideal of $R$ such that $I \cap R_{e}$ is nilpotent, then $I$ is nilpotent.

Throughout, rings and algebras are associative with an identity element 1. All groups are denoted multiplicatively and the group identity is denoted by $e$.

Let $G$ be a group. Recall that a ring $R$ is $G$-graded if $R=\bigoplus_{g \in G} R_{g}$ is the direct sum of additive subgroups $R_{g}$ of $R$ with $R_{g} R_{h} \subseteq R_{g h}$ for all $g, h \in G$. It follows that $R_{e}$ is a subring of $R$ containing 1 . An ideal $I$ of $R$ is called homogeneous if $I=\bigoplus_{g \in G} I \cap R_{g}$. The largest homogeneous ideal of $R$ contained in a given ideal $J$ of $R$ is denoted by $J_{G}$.

The proof of the theorem is based on the following three lemmas:

Lemma 1 [1]. If $I$ is a homogeneous ideal of a ring $R$ graded by a finite group and $I \cap R_{e}$ is nilpotent, then $I$ is nilpotent.

Lemma 2. If $A$ is an F-algebra over a field of characteristic $p>0$, then every ideal $I$ of the group algebra $A[G]$ of a finite p-group $G$ such that $I \cap A=0$ is nilpotent.

Proof. Let $\pi: A[G] \rightarrow A$ be the augmentation homomorphism, i.e. $\pi\left(\sum a_{g} g\right)=$ $\sum a_{g}$. It is well known that $(\operatorname{ker} \pi)^{n}=0$, where $n=|G|$. Hence for every $a_{1}, \ldots, a_{n} \in I,\left(a_{1}-\pi\left(a_{1}\right)\right) \cdots\left(a_{n}-\pi\left(a_{n}\right)\right)=0$. On the other hand, since $I$

Received by the editors April 24, 1989.

1980 Mathematics Subject Classification (1985 Revision). Primary 16A03; Secondary 16A22.

Research supported by Hungarian National Foundation for Scientific Research Grant No. 1813. 
is an ideal, $\left(a_{1}-\pi\left(a_{1}\right)\right) \cdots\left(a_{n}-\pi\left(a_{n}\right)\right)=i \pm \pi\left(a_{1}\right) \cdots \pi\left(a_{n}\right)$ for some $i \in I$. Thus $\pi\left(a_{1}\right) \cdots \pi\left(a_{n}\right) \in I \cap A=0$, which means that $(\pi(I))^{n}=0$. Now $I \subseteq$ $\pi(I)+\operatorname{ker} \pi$ and $(\pi(I)+\operatorname{ker} \pi)^{n} \subseteq \operatorname{ker} \pi$, so $I$ is nilpotent.

Lemma 3. Let $A=\bigoplus_{h \in H} A_{h}$ be a ring graded by an abelian group $H$ and let $f: A \rightarrow A[H]$ be the ring homomorphism of $A$ into the group ring $A[H]$ defined by $f\left(\sum a_{h}\right)=\sum a_{h} h$, where $a_{h} \in A_{h}$.

(i) If $I$ is an ideal of $A$, then the ideal $J$ of $A[H]$ generated by $f(I)$ is equal to $\sum_{g \in H} f(I) g$;

(ii) If $I_{G}=0$, then $J \cap A=0$.

Proof. (i). It suffices to prove that $\sum_{g \in G} f(I) g$ is an ideal of $A[H]$, and for this, since $H$ is abelian, it is enough to check that if $a \in A_{x}$, then $f(I) a \subseteq$ $\sum_{g \in H} f(I) g$ and $a f(I) \subseteq \sum_{g \in H} f(I) g$. Observe that $f(I) a x=f(I) f(a)=$

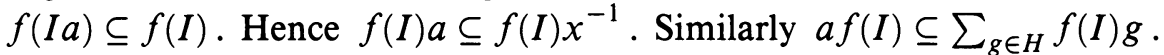

(ii). Suppose that $a \in J \cap A$. By (i), $a$ is a finite sum $\sum_{g} a_{g} g$, with $a_{g} \in$ $f(I)$ and $g \in G$. Each $a_{g}$ is equal to a finite sum $\sum_{h} i_{h, g} h$, where $i_{h, g} \in A_{h}$ and $\sum_{h} i_{h, g} \in I$. Now $\sum_{g} a_{g} g=\sum_{g}\left(\sum_{h} i_{h, g} h\right) g=\sum_{x}\left(\sum_{h g=x} i_{h, g}\right) x \in A$, so for every $e \neq x \in G, \sum_{h g=x} i_{h, g}=0$. This and the fact that $i_{h, g} \in A_{h}$ give that if $h g \neq e$, then $i_{h, g}=0$. Thus $a_{g}=i_{g^{-1}, g} g$. Now $i_{g^{-1}, g} \in I_{G}=0$, so for all $g, a_{g}=0$. This gives $a=0$.

Proof of the theorem. We proceed by induction on $|G|$. Let $|G|=p$. Passing, if necessary, to the factor algebra $R / I_{G}$, one can assume that $I_{G}=0$. Now Lemmas 2 and 3 give immediately that $I$ is nilpotent. If $|G|>p$, then $G$, being a $p$-group, contains a nontrivial normal subgroup $H$. Let $T=\bigoplus_{h \in H} R_{h}$. Obviously $T$ is an $H$-graded algebra, so the induction assumption applied to $T$ gives that $I \cap T$ is nilpotent. Now $R$ has a natural structure of $G / H$ graded algebra with the identity component equal to $T$. Hence the induction assumption gives that $I$ is nilpotent.

Corollary 1 (cf. [2]). Let $A$ be an algebra over a field $F$ of characteristic $p>0$ and let $F \subseteq K=F(a)$ be a field extension with $a^{p^{n}} \in F$ for some $n$. Then every ideal $I$ of $A \otimes_{F} K$ such that $A \cap I=0$ is nilpotent.

Proof. Without loss of generality one can assume that $n$ is a minimal integer with $a^{p^{n}} \in F$. Then $(K: F)=p^{n}$ and $R=A \otimes_{F} K$ can be treated as an algebra graded by the cyclic group $\langle g\rangle$ of order $p^{n}$ with homogeneous components $R_{g^{k}}=A \otimes a^{k}, k=0,1, \ldots, p^{n}-1$. Now the corollary is an immediate consequence of the theorem.

As an immediate consequence of the theorem one also gets

Corollary 2. If $R$ is that of the theorem and $R_{e}$ is Jacobson semisimple, then the Jacobson radical of $R$ is equal to the largest ideal of $R$ not intersecting $R_{e}$.

Of course Corollary 2 is valid for many other radicals as well.

Lemma 2 (so also the theorem) does not hold if $I$ is a one-sided ideal. 
Example. Let $A$ be the algebra of all $2 \times 2$-matrices over a field $F$ of characteristic 2 and let $G=\{e, g\}$ be a group of order 2 . Observe that

$$
I=\left\{\left(\begin{array}{ll}
a & c \\
b & d
\end{array}\right) e+\left(\begin{array}{ll}
c & a \\
d & b
\end{array}\right) g \mid a, b, c, d \in F\right\}
$$

is a left ideal of $A[G]$ such that $I \cap A=0$. Now

$$
\pi(I)=\left\{\left(\begin{array}{ll}
x & x \\
y & y
\end{array}\right) \mid x, y \in F\right\}
$$

so

$$
(\pi(I))^{2}=\pi(I) .
$$

This shows that $I$ is not nilpotent.

\section{REFERENCES}

1. M. Cohen and L. Rowen, Group graded rings, Comm. Algebra 11 (1983), 1253-1270.

2. J. Krempa, On semisimplicity of tensor products, Ring Theory (Proc. Antwerp Conf., Univ. Antwerp, Antwerp, 1978), pp. 105-122; Lectures Notes in Pure and Appl. Math., no. 51, Dekker, New York, 1979.

Institute of Mathematics, University of Warsaw, PKiN, 00-901 Warsaw, Poland

Current address: Institute of Mathematics, University of Warsaw, 00-913 Warsaw 59, Banacha 2, Poland 